\title{
Analysis of Mass Transport Processes in the Anodic Porous Transport Layer in PEM Water Electrolysers
}

\author{
A. Zinser ${ }^{\mathrm{a}}$, G. Papakonstantinou ${ }^{\mathrm{b}}$, K. Sundmacher ${ }^{\mathrm{a}, \mathrm{b}, *}$ \\ ${ }^{a}$ Otto-von-Guericke University, Universitätsplatz 2, 39106 Magdeburg, Germany \\ ${ }^{b}$ Max Planck Institute for Dynamics of Complex Technical Systems, Sandtorstraße 1, 39106 Magdeburg, Germany
}

\begin{abstract}
In this contribution, the transport processes in the anodic porous transport layer (A-PTL) in proton exchange membrane (PEM) water electrolysis are studied in detail. A spatially distributed model of the transport processes in the A-PTL is derived and implemented, which is able to predict stationary saturation profiles and to detect drying-out behaviour at the anodic catalyst layer. The influence of the material properties on the transport limitations is analysed in order to avoid a drying-out of the system. The analysis showed that a suitable PTL material should have a porosity of $\varepsilon_{0}>0.6$, a permeability of $k_{0}>10^{-14} \mathrm{~m}^{2}$, and a contact angle of $\theta<45^{\circ}$. It is also shown that a sufficiently high water saturation in the channel of $S_{\mathrm{ch}}^{\mathrm{L}}>0.8$ is crucial for the transport behaviour. Additionally, the bulk material properties of a Ti sintered fibre PTL are determined experimentally $\left(\varepsilon_{0}=0.75, k_{0}=6.4 \times 10^{-14} \mathrm{~m}^{2}, \theta=30^{\circ}\right)$.
\end{abstract}

Keywords: PEM Water Electrolysis, Anodic Porous Transport Layer, Multiphase Mass Transport

\section{Introduction}

Water splitting using proton exchange membrane (PEM) electrolysis is seen as a key technology for chemical storage of renewable energy of fluctuating and intermittent character [1]. In order to scale-up and optimize the PEM water electrolysis technology the understanding of the transport phenomena is essential. Beside of the experimental investigation of the PEM water electrolysis an important tool for the understanding and the prediction of the process behaviour is the mathematical modelling and simulation. However, compared to PEM fuel cell systems not many models have been reported for the PEM water electrolysis. Rahim etal. 2] give an overview of published models of the PEM water

\footnotetext{
* Corresponding author

Email address: sundmacher@mpi-magdeburg.mpg.de (K. Sundmacher)
}

electrolysis.

The general structure of a PEM water electrolysis cell is depicted in Fig. 1. The anode channel A-CH provides the reactant water and collects the produced oxygen while the cathode channel $\mathrm{C}-\mathrm{CH}$ collects the desired product hydrogen. The membrane (MEM) separates the anode side from the cathode side and lets only the protons pass through. The catalyst layers A-CL and C-CL enable the two electrochemical half-reactions

$$
\begin{aligned}
\mathrm{H}_{2} \mathrm{O} & \rightleftharpoons \frac{1}{2} \mathrm{O}_{2}+2 \mathrm{H}^{+}+2 \mathrm{e}^{-}, \\
2 \mathrm{H}^{+}+2 \mathrm{e}^{-} & \rightleftharpoons \mathrm{H}_{2} .
\end{aligned}
$$

The electrodes of the system are designed as porous transport layers (PTL). Beside of the supply of the electrical current the PTLs must be permeable in order to ensure the mass transport between the channel and the catalyst layers.

September 3, 2019 


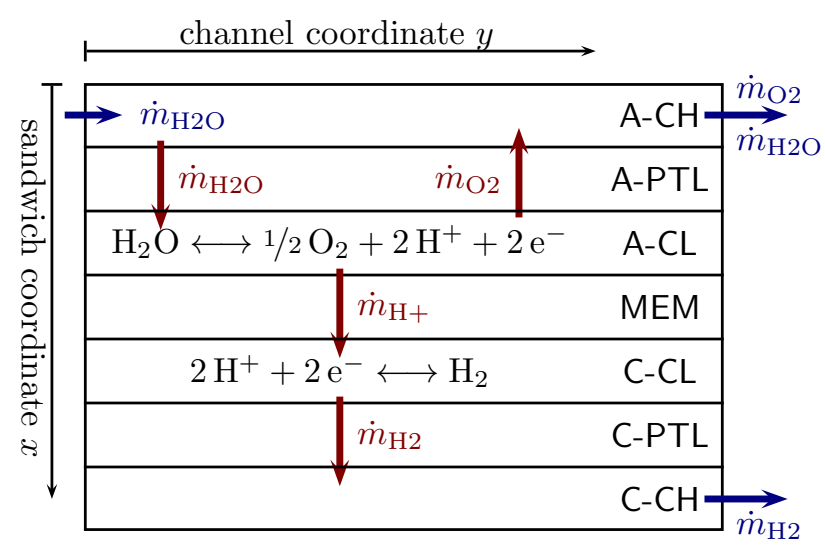

Figure 1: Schematic representation of the different layers in a PEM electrolyser, the mass flows between the layers and the electrochemical reactions in the system.

Spatially lumped models of PEM electrolyser cells are useful tools for the prediction of general behaviour, for studying the influence of system parameters, or for exploring control strategies. These models consider mostly the porous transport layers on the anode and the cathode side, as well as the membrane while the electrochemical reactions are modelled in the interfaces between PTL and MEM. Awasthi et al. [3] implemented a dynamic model in MATLAB/Simulink for the prediction of the cell voltage and validated it using experimental data. Abdin et al. 4] presented a linked modular model of the different layers as a tool for understanding the influence of the cell components on the overall behaviour of the system. Yigit and Selamet [5] propose a system model which includes beside of the PEM electrolyser cell also auxiliary units such as pump, fan and tanks. This allows the study of the overall system behaviour with regard to dynamic changes of the operating current density.

Apart from the spatially lumped models of the overall system, some three-dimensional models of the fluid flow in the channels or in the PTL are available. These models are typically implemented and solved using computational fluid dynamics (CFD) software. Nie and Chen [6] investigate the flow field in a square channel structure. They found that the results from a single phase flow simulation cannot be linearly extrapolated to a two-phase flow. Olesen et al. 7] analyse the maldistribution of the flow on a planar-circular flowfield and its impact on the temperature distribution. Arbabi et al. [8] employ a volume-of-fluid (VOF) method in order to investigate the propagation of oxygen bubbles in the PTL. Lafmejani et al. 9] use a VOF model to predict a Taylor flow in the micro-channels and confirm the simulated results experimentally.

Between the spatially lumped and the fully three-dimensional CFD models some one- and twodimensional models are available to study internal behaviours and effects of the system. The coordinates in a PEM electrolyser cell can be divided into a channel coordinate and a sandwich coordinate, see also Fig. 1] Such an along-the-channel model is presented by Kim et al. [10] which enables the spatially distributed prediction of concentrations, current density and temperature along the channel. Aubras et al. [11] developed a twodimensional model along the sandwich coordinate and predict the transition point between the not coalesced bubble regime and the coalesced bubble regime of the oxygen bubbles. Ojong et al. [12] describe a fully coupled two-dimensional model along both, the channel and the sandwich coordinates. They show the effects of several process parameters on the mass transport limitations. NouriKhorasani etal. 13] employ a one-dimensional model along the sandwich coordinate in order to study formation, growth, and stability of the oxygen bubbles in the A-PTL. Han et al. [14, 15] developed a one-dimensional model along the sandwich coordinate and analyse the influence of the material properties on the mass transport through the A-PTL. They found that increasing the porosity and decreasing the contact angle improves the performance of a PEM electrolysis cell.

The aim of this contribution is to study the transport processes in the anodic porous transport layer 
(A-PTL) and to analyse systematically the influence of the bulk material properties of the A-PTL on the 2-phase flow transport process . Therefore, a spatially distributed mathematical model of the counter-current mass transport problem is derived and discretised. This model is able to predict the steady-state behaviour of the system or to detect a drying-out phenomena at the catalyst layer, dependent on the process parameters and material properties of the A-PTL. Such drying-out phenomena have been observed experimentally by Immerz etal. 16] at low water flow rates and result in an increase of the ohmic resistance. For each set of material properties, i. e. porosity $\varepsilon_{0}$, contact angle $\theta$, and permeability $k_{0}$, a critical current density $i_{\text {crit }}$ can be computed as function of the water saturation in the channel. This critical current density separates the two regimes where a stationary operation of the PEM electrolyser cell occurs and where a drying-out of the catalyst layer is possible.

The material properties of an A-PTL based on Ti sintered fiber is characterised experimentally. The experimentally obtained values are compared with published material properties. A further systematic study was performed that investigates the influence of the mentioned material properties of the A-PTL in detail.

\section{Model derivation}

In this section, a mathematical model of the transport process in the A-PTL is derived. The A-PTL connects the anodic channel with the anodic catalyst layer where the electrochemical splitting of water into oxygen, protons, and electrons takes place, see Eq. (1a). The water has to be transported from the channel to the catalyst where it is consumed and the produced oxygen has to be transported back to the channel in a countercurrent manner. A schematic representation of the structure of this three-phase transport problem is depicted in Fig. 2(a).
In order to derive a mathematical model of this counter-current flow, the following assumptions on the systems are made:

- the transport layer, i. e. the solid phase is immobile,

- the water occurs as pure liquid and is incompressible,

- the oxygen is only in the gas phase and it is described by the ideal gas law,

- the system is assumed to be isothermal.

Thus, for the description of the two fluid phases the following properties are required in each phase $\pi \in\{\mathrm{G}, \mathrm{L}\}:$

- the saturation $S^{\pi}$,

- the pressure $p^{\pi}$, and

- the phase velocity $v^{\pi}$.

For an overview of the unknown states in this system, see also Fig.2(b)] Therefore, for the prediction of the behaviour of the system six equations are required that connect these six dynamic states with each other.

\subsection{Gas phase mass balance}

In the first step, the mass balance of the gas phase is considered. The total mass balance of the gas phase in $1+1$ dimensions is given by $\partial_{t} \rho^{\mathrm{G}}=-\partial_{x}\left(v^{\mathrm{G}} \rho^{\mathrm{G}}\right)$ where $\rho^{\mathrm{G}}$ refers to the density of the gas phase. Assuming the ideal gas law and the fact that the gas phase consists of pure oxygen, the density of the gas phase can be correlated with the gas pressure via $\rho^{\mathrm{G}} R T=\tilde{M}_{\mathrm{O} 2} p^{\mathrm{G}}$ with the molar mass of oxygen $\tilde{M}_{\mathrm{O} 2}$, the gas constant $R$, and the temperature $T$. Assuming isothermal conditions, this leads to a partial differential equation (PDE) that describes the pressure in the gas phase,

$$
\frac{\partial p^{\mathrm{G}}}{\partial t}=-\frac{\partial}{\partial x}\left(v^{\mathrm{G}} p^{\mathrm{G}}\right) .
$$




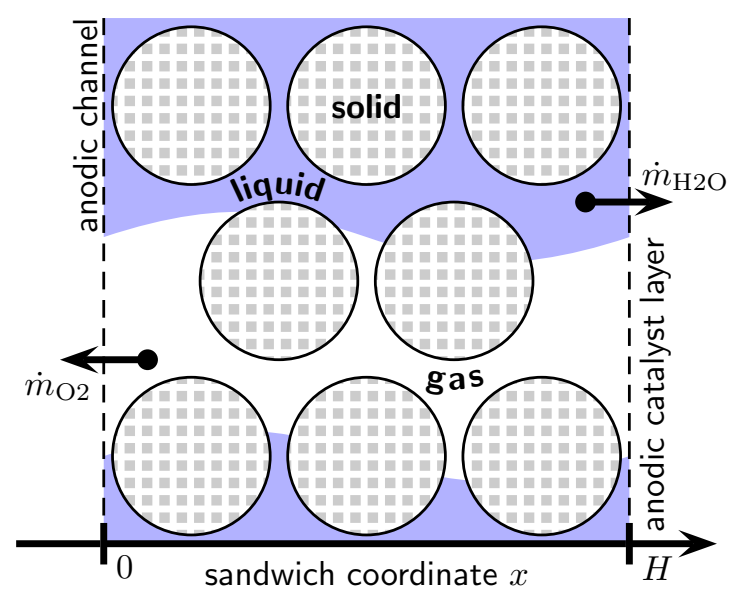

(a) Three-phase structure.

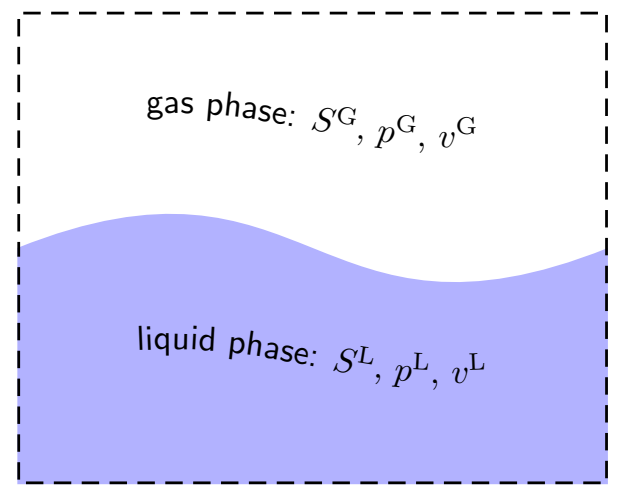

(b) Two-phase model.

Figure 2: (a) Three-phase structure of the anodic porous transport layer (A-PTL). (b) Overview of the dynamic states in order to describe the transport process in the A-PTL: saturation $S^{\pi}$, pressure $p^{\pi}$, and velocity $v^{\pi}$ for each phase $\pi \in\{\mathrm{G}, \mathrm{L}\}$.

\subsection{Liquid phase mass balance}

In the second step, a dynamic equation for the saturation of the liquid phase is derived. For this purpose, some definitions have to be stated. Firstly, the partial phase density of phase $\pi$ is defined by $\tilde{\rho}^{\pi}=m^{\pi} / V$ where the volume of the layer $V$ consists of the partial volumes of the three phases, $V=V^{\mathrm{G}}+V^{\mathrm{L}}+V^{\mathrm{S}}$. The volume fraction $\varepsilon^{\pi}$ of phase $\pi$ is defined by $\varepsilon^{\pi}=V^{\pi} / V$ and, by definition it satisfies the closing condition $\varepsilon^{\mathrm{G}}+\varepsilon^{\mathrm{L}}+\varepsilon^{\mathrm{S}}=1$. Additionally, the void volume $V_{0}$ and the void fraction $\varepsilon_{0}$, which refer to the fluid phases, are defined by $V_{0}=V^{\mathrm{G}}+V^{\mathrm{L}}$, and $\varepsilon_{0}=\varepsilon^{\mathrm{G}}+\varepsilon^{\mathrm{L}}$, respectively. The saturations of the two fluid phases are defined by $S^{\pi}=V^{\pi} / V_{0}$. Combining the density of phase $\pi$, $\rho^{\pi}=m^{\pi} / V^{\pi}$, with the partial phase density $\tilde{\rho}^{\pi}$, the volume fraction $\varepsilon^{\pi}$, and the saturation $S^{\pi}$, leads to the relation

$$
\tilde{\rho}^{\pi}=\varepsilon_{0} \rho^{\pi} S^{\pi}
$$

With the definitions above, a dynamic equation for the liquid saturation $S^{\mathrm{L}}$ can be derived. Therefore, the partial mass of the liquid phase is balanced in the system. With the relation (3) and assuming a constant void fraction $\varepsilon_{0}$ and an incompressible liquid phase, this leads directly to a dynamic equation for the liquid saturation

$$
\frac{\partial S^{\mathrm{L}}}{\partial t}=-\frac{\partial}{\partial x}\left(v^{\mathrm{L}} S^{\mathrm{L}}\right)
$$

Since the saturations of the two fluid phases are directly coupled by the closing condition $S^{\mathrm{G}}+S^{\mathrm{L}}=1$, the gas saturation can be calculated easily from the knowledge of the liquid saturation.

\subsection{Flow field}

The flow field of fluids in porous media can be described by Darcy's law, which connects the velocity of a phase with the pressure gradient [17]. For a fluid phase $\pi \in\{\mathrm{G}, \mathrm{L}\}$, it is given as

$$
v^{\pi}=-\frac{k^{\pi}}{\eta^{\pi}} \frac{\partial p^{\pi}}{\partial x}
$$

where $k^{\pi}$ refers to the permeabilities of the two phases and $\eta^{\pi}$ is the corresponding viscosity. Since each phase consists of a pure substance, the phase viscosities are identical with the viscosities of the substances, i.e. $\eta^{\mathrm{G}}=\eta_{\mathrm{O} 2}$, and $\eta^{\mathrm{L}}=\eta_{\mathrm{H} 2 \mathrm{O}}$, respectively. The permeabilities $k^{\pi}=k_{0} k_{\mathrm{r}}^{\pi}$ consists of the absolute permeability $k_{0}$ and the relative permeabilities $k_{\mathrm{r}}^{\pi}$, which can be correlated by a power 
law approach with the saturation of the particular phase [18]. This leads to the expression

$$
k^{\pi}=k_{0}\left(S^{\pi}\right)^{3}
$$

\subsection{Capillary pressure}

In order to close the system of equations, an expression for the liquid pressure $p^{\mathrm{L}}$ is still required. The connection between the pressures of the liquid and the gaseous phase is given by the capillary pressure $p_{\mathrm{C}}=p^{\mathrm{G}}-p^{\mathrm{L}}$. A common relation for the capillary pressure $p_{\mathrm{C}}$ was derived by Leverett [19] and is given by

$$
p_{\mathrm{C}}=\sigma \cos \theta \sqrt{\frac{\varepsilon_{0}}{k_{0}}} J\left(S^{\mathrm{L}}\right),
$$

with the surface tension $\sigma$, the contact angle $\theta$, the porosity $\varepsilon_{0}$, and the permeability $k_{0}$. For hydrophilic media, i. e. for $\theta<90^{\circ}$, the Leverett function $J\left(S^{\mathrm{L}}\right)$ can be stated as follows [20]

$$
\begin{array}{r}
J\left(S^{\mathrm{L}}\right)=1.417\left(1-S^{\mathrm{L}}\right)-2.12\left(1-S^{\mathrm{L}}\right)^{2} \\
+1.263\left(1-S^{\mathrm{L}}\right)^{3} .
\end{array}
$$

In this work, the capillary pressure is calculated using the Leverett function in accordance with other works in the area of PEM electrolysis 14, 15, 21, 22, 23]. However, it should be noted that the Leverett function was originally developed for the prediction of capillary behaviour of unconsolidated sands in the past [19] and there are also several other empirical correlations for the capillary pressure available such as the Brooks-Corey model [20, 24] or the van Genuchten model [25]. A comparison of the Leverett function and the Brooks-Corey model at the example of a PEM fuel cell system was done by Zamel and Li [20]. They observed minor differences in the liquid water saturation while the overall trend was similar for both models.

\subsection{Boundary conditions}

A summary of the model equations is given in Tab. 1. Combining the equations for the phase velocities with the two mass balance equations, it can be seen that the two PDEs are of second order in space. This means that the system requires also two boundary constraints for each phase.

On the interface between the PTL and the catalyst layer, the mass flow densities of both phases are directly coupled with the production rate of the electrochemical reaction. Hence, the mass flow density of the liquid phase through the interface to the catalyst layer is fixed by

$$
\left.v^{\mathrm{L}} S^{\mathrm{L}}\right|_{x=H}=\frac{\tilde{M}_{\mathrm{H} 2 \mathrm{O}}}{\varepsilon_{0} \rho_{\mathrm{H} 2 \mathrm{O}}} \frac{i_{\mathrm{el}}}{2 F} .
$$

Analogously, the mass flow of the gas phase from the catalyst layer to the PTL is also coupled to the electrochemical reaction, i. e.

$$
\left.v^{\mathrm{G}} p^{\mathrm{G}}\right|_{x=H}=-\frac{R T}{\varepsilon_{0} S^{\mathrm{G}}} \frac{i_{\mathrm{el}}}{F} .
$$

On the side of the interface between the channel and the PTL, the pressure of the gas phase is coupled with the channel pressure $p_{\text {ch }}$ according to

$$
\left.p^{\mathrm{G}}\right|_{x=0}=p_{\text {ch }}+p_{\mathrm{C}}
$$

and the inflow of the liquid phase into the PTL is also coupled with the water consumption due to the electrochemical reaction, i. e.

$$
\left.v^{\mathrm{L}} S^{\mathrm{L}}\right|_{x=0}=\frac{\tilde{M}_{\mathrm{H} 2 \mathrm{O}}}{\varepsilon_{0} \rho_{\mathrm{H} 2 \mathrm{O}}} \frac{i_{\mathrm{el}}}{2 F} .
$$

\section{Discretisation}

In order to solve the partial differential equations of the model, Tab. 1, it is discretised along the spatial coordinate using the finite volume method [26]. Therefore, the domain of the spatial coordinate $\Omega=[0, H]$ is divided into $N$ cells $\Omega_{i}=\left[x_{i-\frac{1}{2}}, x_{i+\frac{1}{2}}\right]$ for all $i=1 \ldots N$ such that 
Table 1: Summary of the model equations.

\begin{tabular}{|c|c|c|}
\hline$S^{\mathrm{G}}=1-S^{\mathrm{L}}$ & $\frac{\partial p^{\mathrm{G}}}{\partial t}=-\frac{\partial}{\partial x}\left(v^{\mathrm{G}} p^{\mathrm{G}}\right)$ & $v^{\mathrm{G}}=-\frac{k^{\mathrm{G}}}{\eta^{\mathrm{G}}} \frac{\partial p^{\mathrm{G}}}{\partial x}$ \\
\hline$\frac{\partial S^{\mathrm{L}}}{\partial t}=-\frac{\partial}{\partial x}\left(v^{\mathrm{L}} S^{\mathrm{L}}\right)$ & $p^{\mathrm{L}}=p^{\mathrm{G}}-p_{\mathrm{C}}$ & $v^{\mathrm{L}}=-\frac{k^{\mathrm{L}}}{\eta^{\mathrm{L}}} \frac{\partial p^{\mathrm{L}}}{\partial x}$ \\
\hline
\end{tabular}

$\bigcup_{i=1}^{N} \Omega_{i}=\Omega$. The centres of the discrete cells are defined by $x_{i}=\left(x_{i-\frac{1}{2}}+x_{i+\frac{1}{2}}\right) / 2$, and the widths $\Delta x_{i}=x_{i+\frac{1}{2}}-x_{i-\frac{1}{2}}$, respectively. Note, that the whole-numbered indices refer to the centre of the cells while non-whole-numbered indices refer to the boundary of the cells. Within a cell $\Omega_{i}$, the value of the spatially distributed states are approximated by the integral mean value which follows from the mean value theorem.

The equations for the saturations $S^{\pi}$ and the pressures $p^{\pi}$ are discretised by integration over a cell $\Omega_{i}$. For the discretisation procedure of the accumulation terms the Leibniz integral rule is applied, and the discretisation of the convective transport term the divergence theorem is utilised [27]. This leads to the discretised equations for the saturation

$$
\begin{aligned}
S_{i}^{\mathrm{G}} & =1-S_{i}^{\mathrm{L}} \\
\frac{\mathrm{d} S_{i}^{\mathrm{L}}}{\mathrm{d} t} & =-\frac{1}{\Delta x_{i}}\left(v_{i+\frac{1}{2}}^{\mathrm{L}} S_{i+\frac{1}{2}}^{\mathrm{L}}-v_{i-\frac{1}{2}}^{\mathrm{L}} S_{i-\frac{1}{2}}^{\mathrm{L}}\right),
\end{aligned}
$$

as well as for the pressures

$$
\begin{aligned}
\frac{\mathrm{d} p_{i}^{\mathrm{G}}}{\mathrm{d} t} & =-\frac{1}{\Delta x_{i}}\left(v_{i+\frac{1}{2}}^{\mathrm{G}} p_{\mathrm{i}+\frac{1}{2}}^{\mathrm{G}}-v_{i-\frac{1}{2}}^{\mathrm{G}} p_{\mathrm{i}-\frac{1}{2}}^{\mathrm{G}}\right), \\
p_{i}^{\mathrm{L}} & =p_{i}^{\mathrm{G}}-p_{\mathrm{C}}\left(S_{i}^{\mathrm{L}}\right) .
\end{aligned}
$$

While the saturations and pressures are discretised in the finite volumes, the phase velocities are approximated directly on the boundaries of the cells. This corresponds to the discretisation scheme proposed by Patankar 28]. An overview of the discretisation scheme is shown in Fig. 3 .

Since the phase velocities $v^{\pi}$ have to be discretised on the boundaries of the cells, according to
Darcy's law, also the permeabilities and the pressure gradients have to be approximated on the boundaries on the cells

$$
v_{i+\frac{1}{2}}^{\pi}=-\left.\left.\frac{1}{\eta^{\pi}} k^{\pi}\right|_{x_{i+\frac{1}{2}}} \frac{\partial p^{\pi}}{\partial x}\right|_{x_{+i \frac{1}{2}}} .
$$

The permeabilities on the cell boundaries are approximated as the weighted average of the two neighbouring permeabilities,

$$
\left.k^{\pi}\right|_{x_{i+\frac{1}{2}}}=\frac{k^{\pi}\left(S_{i}^{\pi}\right) \Delta x_{i}+k^{\pi}\left(S_{i+1}^{\pi}\right) \Delta x_{i+1}}{\Delta x_{i}+\Delta x_{i+1}},
$$

which corresponds to a Beam-Warming scheme [26]. The pressure gradients on the boundaries are approximated by the two-point symmetric difference scheme

$$
\left.\frac{\partial p^{\pi}}{\partial x}\right|_{x_{i+\frac{1}{2}}}=2 \frac{p_{i+1}^{\pi}-p_{i}^{\pi}}{\Delta x_{i+1}+\Delta x_{i}}
$$

In order to discretise the partial differential equations, the dynamic states $p^{\mathrm{G}}$ and $S^{\mathrm{L}}$ must be approximated on the boundaries of the cells. This is done by the first order upwind scheme, which considers also the direction of the phase flow, i.e. $f_{i+\frac{1}{2}}=f_{i}$ for positive flow direction, and $f_{i+\frac{1}{2}}=f_{i+1}$ for negative flow direction, respectively. Here, $f$ refers to the two differential dynamic states $f \in\left\{p^{\mathrm{G}}, S^{\mathrm{L}}\right\}$.

Up to now, the numerical scheme is developed for an arbitrary inner control volume $\Omega_{i}$. Since the numerical approximation of the pressure gradient, Eq. (15), is not applicable on the first and the last cell boundary, the boundary conditions of the 


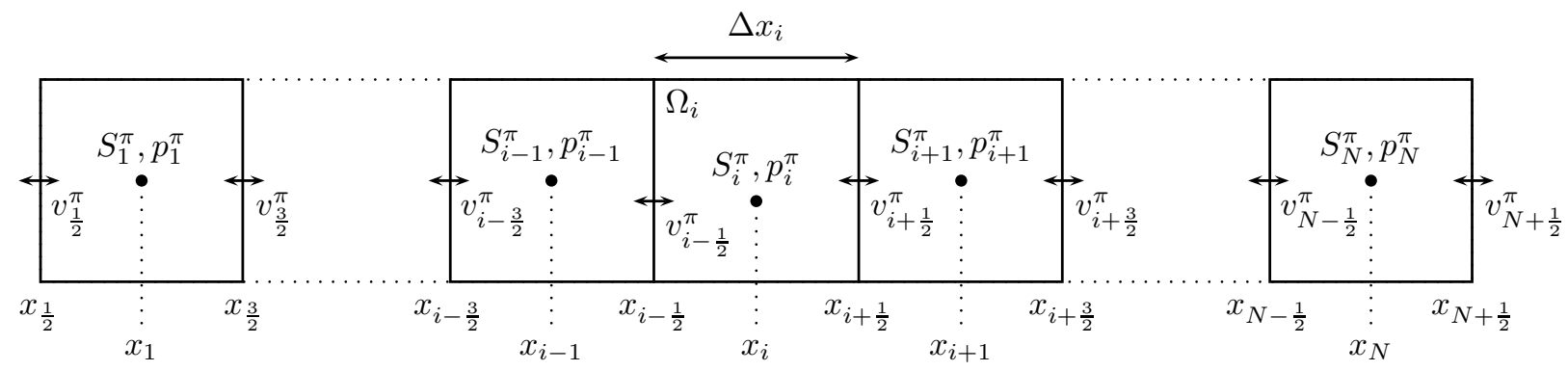

Figure 3: Overview of the discretisation scheme.

problem are incorporated here. The liquid phase boundary conditions, Eq. (11) and (8), yield in combination with Darcy's law, Eq. (5), to the following expressions for the pressure gradients at inlet

$$
\left.\frac{\partial p^{\mathrm{L}}}{\partial x}\right|_{x_{\frac{1}{2}}}=-\frac{\eta_{\mathrm{H} 2 \mathrm{O}} \tilde{M}_{\mathrm{H} 2 \mathrm{O}}}{k_{1}^{\mathrm{L}} S_{1}^{\mathrm{L}} \varepsilon_{0} \rho_{\mathrm{H} 2 \mathrm{O}}} \frac{i_{\mathrm{el}}}{2 F},
$$

and outlet

$$
\left.\frac{\partial p^{\mathrm{L}}}{\partial x}\right|_{x_{N+\frac{1}{2}}}=-\frac{\eta_{\mathrm{H} 2 \mathrm{O}} \tilde{M}_{\mathrm{H} 2 \mathrm{O}}}{k_{N}^{\mathrm{L}} S_{N}^{\mathrm{L}} \varepsilon_{0} \rho_{\mathrm{H} 2 \mathrm{O}}} \frac{i_{\mathrm{el}}}{2 F},
$$

respectively. At the interface between the channel and the PTL, the gas pressure is given by Eq. (10), which leads to the pressure gradient

$$
\left.\frac{\partial p^{\mathrm{G}}}{\partial x}\right|_{x_{\frac{1}{2}}}=\frac{p_{1}^{\mathrm{G}}-\left[p_{\mathrm{ch}}+p_{\mathrm{C}}\left(S_{1}^{\mathrm{L}}\right)\right]}{\Delta x_{1} / 2} .
$$

Analogously to the boundary conditions of the liquid phase, the boundary condition of the gas phase at the interface to the catalyst layer, Eq. (9), leads to

$$
\left.\frac{\partial p^{\mathrm{G}}}{\partial x}\right|_{x_{N+\frac{1}{2}}}=\frac{\eta_{\mathrm{O} 2} R T}{k_{N}^{\mathrm{G}} S_{N}^{\mathrm{G}} \varepsilon_{0} p_{N}^{\mathrm{G}}} \frac{i_{\mathrm{el}}}{F} .
$$

\section{Simulation}

The simulations in this work were performed on a system with the following configuration:

Hardware: Intel ${ }^{\circledR}$ Core $^{\mathrm{TM}}$ i5-6500 (6 MiB Cache, $3.2 \mathrm{GHz}), 8 \mathrm{GiB}$ RAM.
Operating System: Microsoft Windows 7 Professional, Version 6.1 (Build 7601, SP 1).

Software: MATLAB Version 9.0.0.341360 (R2016a), Java 1.7.0_60-b19 with Oracle Corporation Java HotSpot ${ }^{\mathrm{TM}}$ 64-Bit Server VM mixed mode.

The proposed model of the transport processes has two spatially distributed dynamic states, namely the liquid saturation $S^{\mathrm{L}}$ and the gas pressure $p^{\mathrm{G}}$. The four remaining states are of algebraic nature and are given explicitly. Therefore, a system of $2 N$ ordinary differential equations has to be solved. This is done in MATLAB using the solver ode15s [29].

In order to optimize the computational performance of the ode15s solver, the structural Jacobian matrix is provided to the ODE solver. The structural Jacobian is of the dimension $2 N \times 2 N$ and consists of $2 \times 2$ tridiagonal $N \times N$ submatrices.

Within this work, a discretisation of $N=100$ control volumes is applied. In order to determine whether the number of control volumes is sufficient the discretisation was varied and compared against a high-resolution simulation with $N=10^{4}$ which was assumed to be the exact solution. The results of the gas pressure $p^{\mathrm{G}}$ reach an error of less than $1 \%$ for discretisations of $N>60$ and the results for the liquid saturation $S^{\mathrm{L}}$ are less than $0.1 \%$ for $N>60$. Therefore, the selected discretisation of $N=100$ 
seems to be a reasonable compromise between accuracy and computational effort.

Since the boundary conditions for the liquid saturation $S^{\mathrm{L}}$, Eq. (8) and (11), are both set equal to the mass flux due to the electrochemical reaction in the catalyst layer, which holds only for the steadystate behaviour of the process, the derived model is only suitable for the analysis of the transport process in its stationary point. This leads to the effect that the mean saturation $\bar{S}^{\mathrm{L}}(t)=\frac{1}{H} \int_{0}^{H} S^{\mathrm{L}}(t, x) \mathrm{d} x$ is constant during the simulation time and depends only on the initial profile of the saturation $S^{\mathrm{L}}(t=0, x)$. Therefore, the channel saturation $S_{\mathrm{ch}}^{\mathrm{L}}$ is given a posteriori as $S_{\mathrm{ch}}^{\mathrm{L}}=S^{\mathrm{L}}(t \rightarrow \infty, x=0)$.

Additionally, since the saturations $S^{\pi}$ occur in the denominators of the boundary conditions, the proposed model does not allow a pure liquid or a pure gas phase in the PTL.

\subsection{Initialisation procedure}

Consistent initial profiles for the liquid saturation $S_{i}^{\mathrm{L}}$ and the gas pressure $p_{i}^{\mathrm{G}}$ have to be assumed in order to solve the ODE system. Beside of the ODE system, the saturations of both phases as well as the pressures of both phases are also coupled by algebraic conditions. For a given mean saturation $\bar{S}^{\mathrm{L}}$ a corresponding initial saturation profile $S^{\mathrm{L}}(0, x)=S_{0}^{\mathrm{L}}(x)$ is constructed according to $S_{0}^{\mathrm{L}}(x)=\bar{S}^{\mathrm{L}}+\Delta S\left(1-\frac{2 x}{H}\right)$ with $\Delta S=\frac{3}{5} \min \left(1-\bar{S}^{\mathrm{L}}, \bar{S}^{\mathrm{L}}\right)$. This construction ensures that $S_{0}^{\mathrm{L}}(x) \in(0,1)$ for all $x$ and $\bar{S}^{\mathrm{L}} \in(0,1)$.

In case of the liquid pressure, also a linear spatial initial profile $p^{\mathrm{L}}(0, x)=p_{0}^{\mathrm{L}}(x)$ is assumed. The inlet pressure is set equal to the channel pressure $p_{\text {ch }}$ and a pressure drop of $10 \%$ through the PTL is assumed. This leads to the initial profile $p_{0}^{\mathrm{L}}(x)=p_{\mathrm{ch}}\left(1-\frac{x}{10 H}\right)$. In order to obtain a consistent initial expression for the gas pressure $p^{\mathrm{G}}(0, x)=p_{0}^{\mathrm{G}}(x)$ the capillary pressure is employed, i. e. $p_{0}^{\mathrm{G}}(x)=p_{0}^{\mathrm{L}}(x)+p_{\mathrm{C}}\left(S_{0}^{\mathrm{L}}(x)\right)$.

\subsection{System parameters}

An overview of the physical constants and the properties of the fluid phases which are required by the model are summarized in Tab. 2. Additionally, a channel pressure of $p_{\mathrm{ch}}=0.12 \mathrm{MPa}$ and a thickness of the PTL of $H=1 \mathrm{~mm}$ is assumed.

In literature, porous transport layers of different materials, e.g. carbon or titanium, as well as different structures, such as sintered powder or fibred felts, are investigated [21, 31, 32, 33, 34]. Nevertheless, the impact of all those materials and structures on the transport behaviour can be characterised by the bulk properties, such as the porosity $\varepsilon_{0}$, the permeability $k_{0}$, and the contact angle $\theta$. For an initial simulation study, the material parameters are chosen according to Lemoine-Nava et al. [21], which are summarized as material \#2 in Tab. 4 . It should be noted, that these parameters originate from a carbon PTL in a fuel cell system. In the later analysis of the material properties it is shown that carbon is not the optimal choice for the A-PTL material in case of electrolysis technology.

\subsection{Stationary profiles}

For the given process parameters, the system was simulated towards its steady-state with an current density of $i_{\mathrm{el}}=0.5 \mathrm{~A} / \mathrm{cm}^{2}$ and a mean saturation of $\bar{S}^{\mathrm{L}}=0.5$. In order to detect the steady-state, the system was simulated up to a time of $10^{5} \mathrm{~s}$, while no significant change of the profile can be detected after $100 \mathrm{~s}$. The typical run-time of such a simulation is approximately $50 \mathrm{~ms}$.

The stationary profiles of the liquid saturation $S^{\mathrm{L}}$, as well as the profiles of the two pressures $p^{\pi}, \pi \in\{\mathrm{G}, \mathrm{L}\}$ are displayed in Fig. 4. In this simulation, a corresponding channel saturation of $S_{\mathrm{ch}}^{\mathrm{L}}=0.75$ was detected and decreases with the sandwich coordinate. In the liquid pressure profile, only very small negative gradient can be seen, while the gas pressure profile has a stronger and positive gradient. 
Table 2: Physical constants and properties of the fluid phases [30]. Note, that the fluid properties $\rho, \eta$ and $\sigma$ are temperaturedependent and the values given here refer to $T=60^{\circ} \mathrm{C}$.

\begin{tabular}{cll}
\hline Symbol & Quantity & Value \\
\hline$F$ & Faraday constant & $96485 \mathrm{C} \mathrm{mol}^{-1}$ \\
$R$ & gas constant & $8.3145 \mathrm{~J} \mathrm{~mol}^{-1} \mathrm{~K}^{-1}$ \\
$\tilde{M}_{\mathrm{H} 2 \mathrm{O}}$ & molar mass of water & $18.015 \mathrm{~g} \mathrm{~mol}^{-1}$ \\
$\rho_{\mathrm{H} 2 \mathrm{O}}$ & liquid density of water & $983.2 \mathrm{~kg} \mathrm{~m}^{-3}$ \\
$\eta_{\mathrm{H} 2 \mathrm{O}}$ & viscosity of water & $469 \mu \mathrm{Pa} \mathrm{s}$ \\
$\eta_{\mathrm{O} 2}$ & viscosity of oxygen & $22.5 \mu \mathrm{Pa} \mathrm{s}$ \\
$\sigma$ & surface tension of water & $66.3 \mathrm{mN} \mathrm{m}^{-1}$ \\
\hline
\end{tabular}
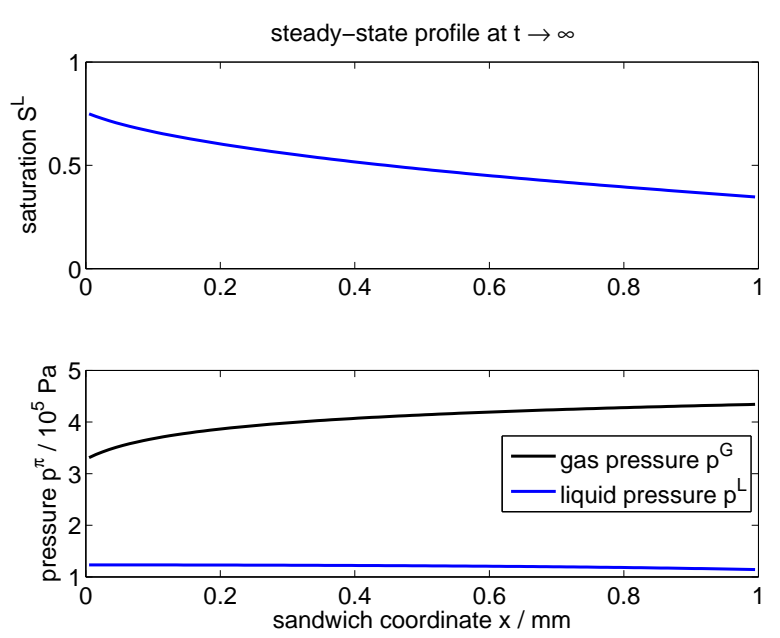

Figure 4: Liquid saturation profile (top) and pressure profiles of both phases (bottom) for a current density of $i_{\mathrm{el}}=$ $0.5 \mathrm{~A} / \mathrm{cm}^{2}$.

\subsection{Drying-out effect}

The simulation is repeated for different current densities. For $i_{\mathrm{el}}=1 \mathrm{~A} / \mathrm{cm}^{2}$, still a steady-state profile is obtained while for $i_{\mathrm{el}}=2 \mathrm{~A} / \mathrm{cm}^{2}$ a dryingout of the catalyst is detected. This means, the saturation at the boundary to the catalyst layer reaches zeros within a finite time, i.e. $\exists t<\infty$ such that $S^{\mathrm{L}}(t, x=H)=0$.

In this case, the drying-out is detected at $t=2.74 \mathrm{~s}$ which is much faster than in a stationary profile where for times up to $t=10^{5} \mathrm{~s}$ no such effect is observed. Fig. 5 shows the stationary profile for $i_{\mathrm{el}}=1 \mathrm{~A} / \mathrm{cm}^{2}$ as well as the profile at the drying-out point for $i_{\mathrm{el}}=2 \mathrm{~A} / \mathrm{cm}^{2}$.
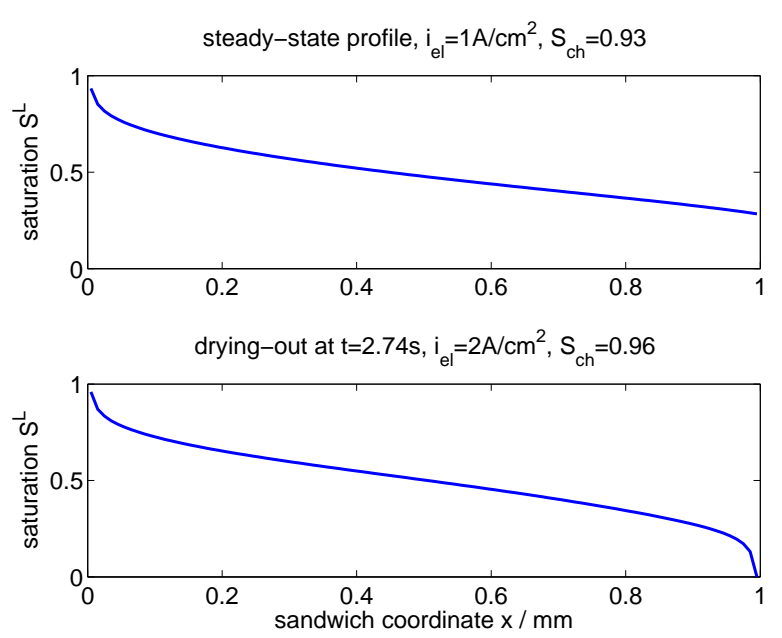

Figure 5: Saturation profiles for different current densities. For $i_{\mathrm{el}}=1 \mathrm{~A} / \mathrm{cm}^{2}$ a stationary profile is reached (top), for $i_{\mathrm{el}}=2 \mathrm{~A} / \mathrm{cm}^{2}$ a drying-out of the catalyst layer is detected in finite time.

\subsection{Critical current density}

As shown in the previous simulations, for higher current densities a drying-out effect may occur. In order to examine this occurrence more detailed, in the following study the current density as well as the mean saturation are varied. Therefore, the intervals $0.08 \mathrm{~A} \mathrm{~cm}^{-2} \leq i_{\mathrm{el}} \leq 12 \mathrm{~A} \mathrm{~cm}^{-2}$, and $0.1 \leq \bar{S}^{\mathrm{L}} \leq 0.99$, respectively, are investigated. For each point in the $\left(i_{\mathrm{el}}, \bar{S}^{\mathrm{L}}\right)$-space the corresponding channel saturation $S_{\text {ch }}^{\mathrm{L}}$ is calculated. Additionally, a critical current density $i_{\mathrm{el}, \text { crit }}$ is defined for a given channel saturation $S_{\mathrm{ch}}^{\mathrm{L}}$, such that the system remains stable for all current densities $i_{\mathrm{el}} \leq i_{\mathrm{el}, \text { crit }}$, or more precisely for all cur- 


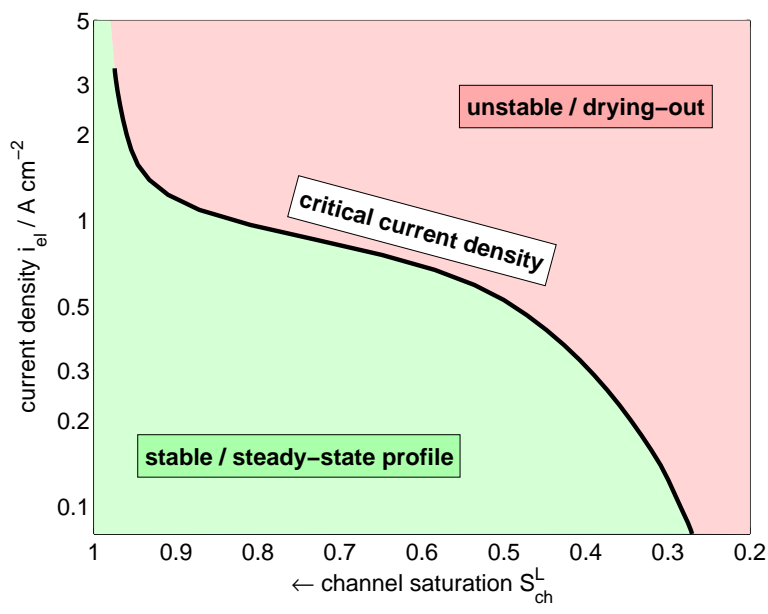

Figure 6: Critical current density $i_{\mathrm{el}, \mathrm{crit}}$ as function of the channel saturation $S_{\mathrm{ch}}^{\mathrm{L}}$ which separates the stable steadystate regime from the unstable drying-out regime.

rent densities $i_{\mathrm{el}} \leq i_{\mathrm{el}, \mathrm{crit}}\left(S_{\mathrm{ch}}^{\mathrm{L}}\right)$ the saturation in the A-PTL satisfies $S^{\mathrm{L}}(t \rightarrow \infty, 0)=S_{\mathrm{ch}}^{\mathrm{L}}$, and $S^{\mathrm{L}}(t \rightarrow \infty, H)>0$.

The critical current density $i_{\text {el,crit }}$ separates the stable regime in which steady-state profiles through the PTL are achieved from the unstable regime where a drying-out of the catalyst layer takes place. Fig. 6] shows the two regimes, and the critical current density as function of the channel saturation $S_{\mathrm{ch}}^{\mathrm{L}}$. It can be seen clearly, that for high channel saturations also high current densities are possible and for decreasing channel saturation, also the maximum allowable current density decreases.

\section{Experimental investigation of material properties of the PTL}

The properties of a typical Ti-PTL assessed in our laboratory were the contact angle $\theta$ and the permeability $k_{0}$. The material used was a Ti sintered fibre media (Ti-Gr 1, Bekipor, Bekaert, Belgium) with a porosity of $\varepsilon=0.75$ and a fibres' diameter of $\delta=35 \mu \mathrm{m}$.

The contact angle was measured with sessile drop technique on an OCA20 device (DataPhysics, Filderstadt, Germany) providing a value of $\theta=30^{\circ}$ with a pre-wetted felt. This value is in good accordance with literature for similar materials [32, 33]. It should be mentioned that for dry material a contact angle of $\theta=90^{\circ}$ was measured which was disregarded because it is not realistic under PEM water electrolysis conditions.

In order to determine the permeability $k_{0}$ only the anodic PTL was placed in the single cell. For more details of the set-up, see e.g. cell C2 in Immerz et al. [35], which was used in flow through mode, i. e. anode to cathode. From Darcy's law, we get

$$
\dot{V}=A v=\frac{A k_{0}}{\eta} \frac{\Delta p}{\Delta x}
$$

where $\dot{V}$ is the volumetric flow rate trough the PTL and $A$ is the active flow through area. The pressure drop through the $\mathrm{Ti}$ felt is given by $\Delta p$ and its thickness is $\Delta x$. The overall PTL has a diameter of $92.85 \mathrm{~mm}$ which corresponds to an active area of $A=6771 \mathrm{~mm}^{2}$.

The permeability is measured in two different configurations. In the first set-up, two PTLs are used which corresponds to a thickness of $\Delta x=$ $2 \mathrm{~mm}$. In the second configuration, one PTL is replaced by a $\mathrm{Ti}$ grid which leads to an active thickness of the layer of $1 \mathrm{~mm}$. The pressure drop was measured with two attached pressure gauges (CPT 6200, 1..16 bar range, WIKA, Germany) close to the inlet and outlet of the anode and cathode, respectively. The pressure drop of the cell without PTLs was also measured and was subtracted from the data in PTLs presence to account for the pressure drop due to tubes.

For the two different configurations a volumetric flow $\dot{V}$ of water was applied and varied on a range of $500 \mathrm{~cm}^{3} / \mathrm{min} \leq \dot{V} \leq 1000 \mathrm{~cm}^{3} / \mathrm{min}$ and the respective pressure drop $\Delta p$ in the system was measured. Darcy's law, Eq. (18), describes the linear correlation between the pressure drop $\Delta p$ and the volumetric flow rate $\dot{V}$. The experimental measurements for the different configurations are depicted 


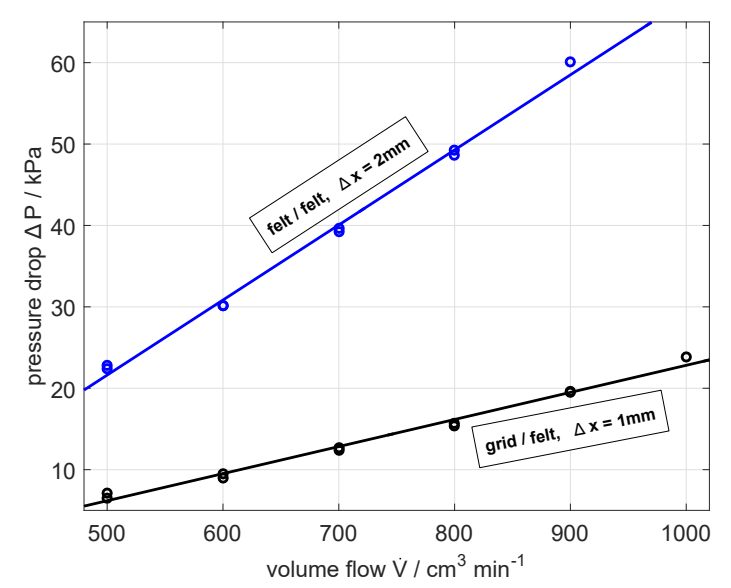

Figure 7: Pressure drop $\Delta p$ through the PTL as function of the volumetric flow rate $\dot{V}$ for the two different configurations: experimental measurements (०) and linear regression $(-)$.

Table 3: Results of the permeability measurements.

\begin{tabular}{ccc}
\hline system & thickness $\Delta x$ & permeability $k_{0}$ \\
\hline grid / felt & $1 \mathrm{~mm}$ & $7.41 \times 10^{-14}$ \\
felt / felt & $2 \mathrm{~mm}$ & $5.35 \times 10^{-14}$ \\
\hline \hline
\end{tabular}

in Fig. 7 as well as the respective linear regressions.

The viscosity of liquid water at ambient temperature $T=20^{\circ} \mathrm{C}$ is given by $\eta_{\mathrm{H} 2 \mathrm{O}}=1002 \mu$ Pas. With that, from the slopes of these regressions the permeability for each configuration is computed. The results are summarized in Tab. 3 and assuming the permeability to be normal distributed one gets an expected value of $k_{0}=6.38 \times 10^{-14} \mathrm{~m}^{2}$. Therefore, the material properties of the Ti felt which is used as A-PTL are summarized as material \#4 in Tab.4.

The prediction of the critical current density as function of the channel saturation is repeated for the determined material properties as well as for a set of different other materials reported in literature. For an overview of the different material properties, see Tab. 4. Therefore, the current density $i_{\mathrm{el}}$ and the mean saturation $\bar{S}^{\mathrm{L}}$ are varied on

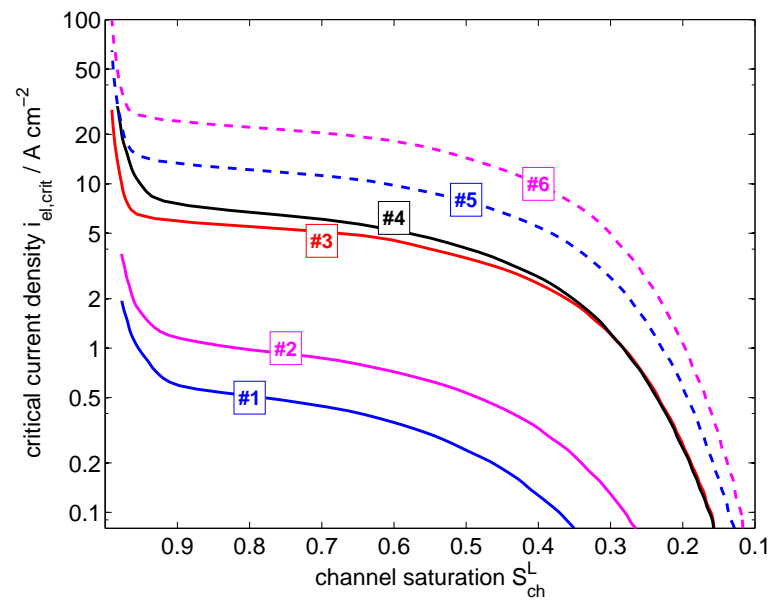

Figure 8: Critical current densities as function of the channel saturation for different material properties. \#1 and \#2 refer to carbon PTLs and \#3 to \#6 refer to different titanium PTLs. \#4 (black) refers to the Ti felt from our lab which was examined experimentally in section 5 See Tab. 4 for a summary of the material properties.

the intervals

$$
\begin{aligned}
0.08 & \leq \frac{i_{\mathrm{el}}}{\mathrm{A} / \mathrm{cm}^{2}} \leq 100, \text { and } \\
0.1 & \leq \bar{S}^{\mathrm{L}} \leq 0.99
\end{aligned}
$$

and the corresponding critical current densities are shown in Fig. 8. For a better distinction of the drying-out regions for the different materials the theoretical upper limit of the current density applied to the system is $100 \mathrm{~A} \mathrm{~cm}^{-2}$, see Eq. (19a). It can be seen that the considered titanium materials allow current densities of clearly more than $10 \mathrm{~A} \mathrm{~cm}^{-2}$ for sufficient high channel saturations. Additionally, it can be seen that the carbon PTLs \#1 and \#2 show relatively small critical current densities. This means that they show drying-out phenomena already at moderate current densities. Therefore, it can be stated that carbon is no suitable material for the A-PTL in PEM water electrolysis technology. 
Table 4: Overview of the material properties of different PTLs.

\begin{tabular}{llcccl}
\hline & & porosity & contact angle & permeability & \\
$\varepsilon_{0}$ & material & $\theta$ & $k_{0} / \mathrm{m}^{2}$ & reference \\
\hline$\# 1$ & carbon & 0.6 & $45^{\circ}$ & $2.1 \times 10^{-16}$ & {$[34]$} \\
$\# 2$ & carbon & 0.6 & $45^{\circ}$ & $2.0 \times 10^{-15}$ & {$[21]$} \\
$\# 3$ & $\mathrm{Ti}$ (sintered) & 0.3 & $30^{\circ}$ & $1.4 \times 10^{-12}$ & {$[32]$} \\
$\# 4$ & $\mathrm{Ti}$ (felts) & 0.75 & $30^{\circ}$ & $6.4 \times 10^{-14}$ & this work (section [5] \\
$\# 5$ & Ti (sintered) & 0.46 & $30^{\circ}$ & $1.9 \times 10^{-12}$ & {$[33]$} \\
$\# 6$ & Ti (homogeneous) & 0.52 & $30^{\circ}$ & $4.5 \times 10^{-12}$ & {$[33]$} \\
\hline
\end{tabular}

\section{Analysis of the influence of the material properties}

In the previous simulations, it was shown that the critical current density and therefore the drying-out behaviour of the anodic PTL strongly depends on the material properties of the transport layer.

In order to investigate the influence of the material properties on the transport behaviour, beside of the current density and the mean saturation, also the material properties are varied. In three studies, the porosity $\varepsilon_{0}$, the permeability $k_{0}$, and the contact angle $\theta$ are varied. In every case, the current density and the mean saturation are varied on the intervals given in Eq. (19).

\subsection{Porosity}

In the first study, the influence of the porosity $\varepsilon_{0}$ is examined. Therefore, the porosity $\varepsilon_{0}$ is varied on the range $0.1 \leq \varepsilon_{0} \leq 0.99$, while the permeability and the contact angle are kept constant according to material \#4 (Tab. 4). For each point in the $\left(i_{\mathrm{el}}, \bar{S}^{\mathrm{L}}, \varepsilon_{0}\right)$-space the corresponding channel saturation $S_{\mathrm{ch}}^{\mathrm{L}}$ and the system behaviour, i.e. steady-state behaviour or drying-out in finite time, are determined. From this information, the critical current density on each point of the $\left(S_{\mathrm{ch}}^{\mathrm{L}}, \varepsilon_{0}\right)$ plane was calculated and the results are displayed in Fig. 9(a) It is shown that high current densities can be sustained for sufficient channel saturation, e.g. $S_{\mathrm{ch}}^{\mathrm{L}} \geq 0.4$, for porosities of $\varepsilon_{0}>0.4$.

\subsection{Permeability}

In the second study, the permeability $k_{0}$ is varied in a logarithmic manner on the interval $10^{-16} \mathrm{~m}^{2} \leq$ $k_{0} \leq 10^{-12} \mathrm{~m}^{2}$ while the porosity and the contact angle are kept constant according to material \#4 (Tab. 4). The critical current density in the $\left(S_{\mathrm{ch}}^{\mathrm{L}}, k_{0}\right)$-space is shown in Fig. 9(b). It can be seen, that especially for high permeabilities the channel saturation has lower influence on the critical current density.

\subsection{Contact angle}

The influence of the contact angle $\theta$ on the transport limitations was also examined. In order to realize the counter-current mass transport of the two phases opposing pressure gradients have to be established in the two phases. Since the capillary pressure as defined in Eq. (7a) is the major property that enables this behaviour one can limit the range for the contact angle to $0^{\circ} \leq \theta \leq 90^{\circ}$. For a contact angle of $\theta=90^{\circ}$ the capillary pressure becomes $p_{\mathrm{C}}=0$ and a counter-current mass transport in two distinct phases becomes impossible. This effect can also be seen in the resulting Fig. 9(c) which shows the critical current density in the $\left(S_{\mathrm{ch}}^{\mathrm{L}}, \theta\right)$ plane. To achieve high current density a contact angle of $\theta<70^{\circ}$ is required for channel saturations $S_{\mathrm{ch}}^{\mathrm{L}}>0.4$.

\section{Conclusion}

A set of model equations that describes the twophase mass transport of water and oxygen through 


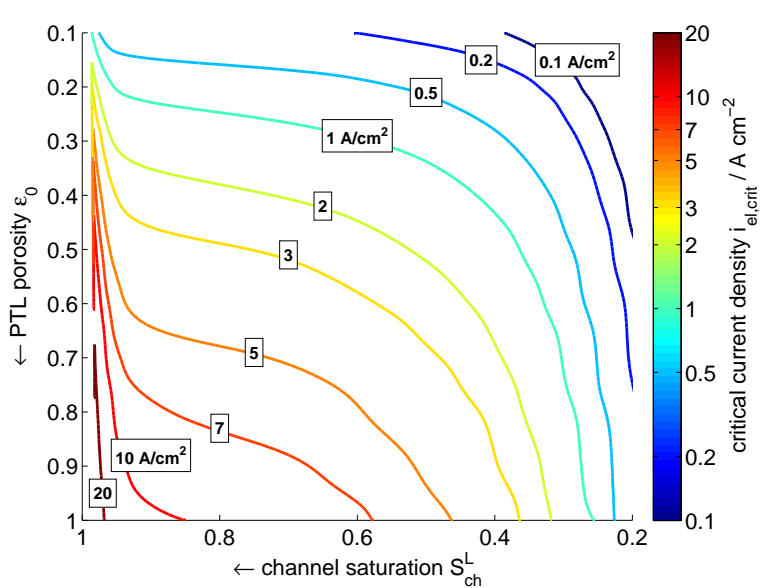

(a) Porosity.

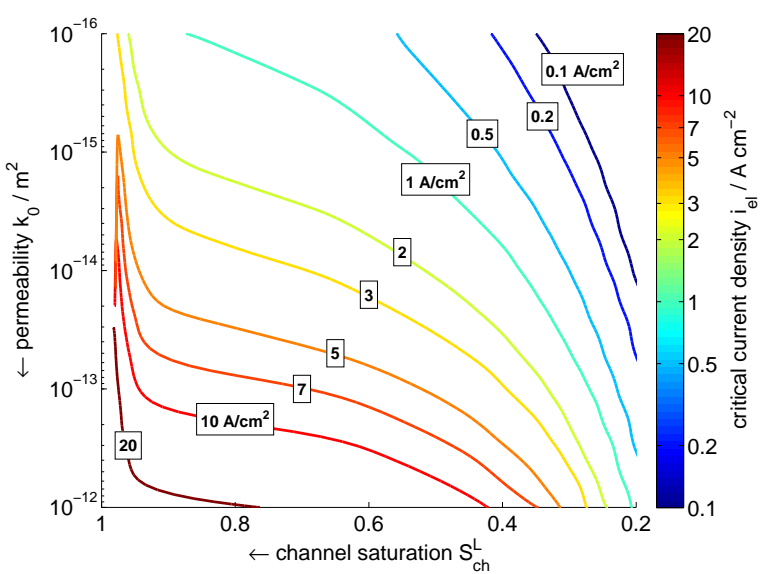

(b) Permeability.

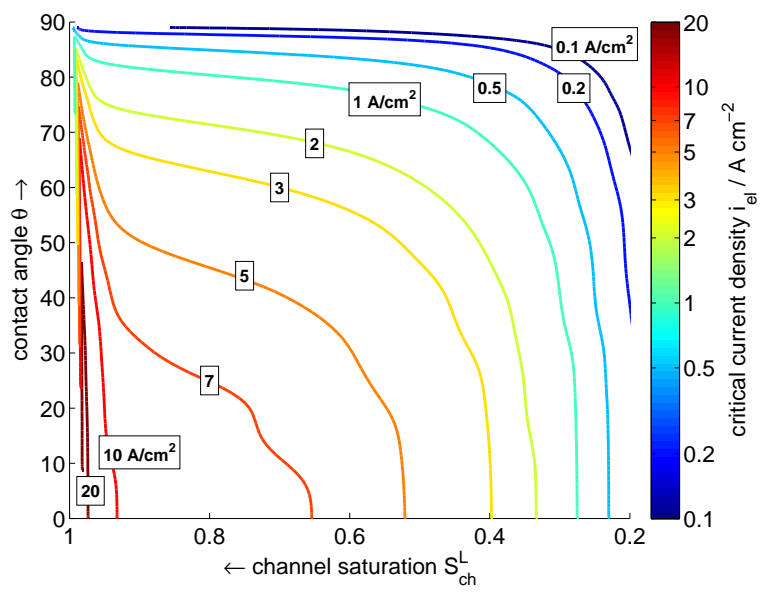

(c) Contact angle.

Figure 9: Critical current density $i_{\mathrm{el}, \mathrm{crit}}$ as function of a material property and the channel saturation $S_{\mathrm{ch}}^{\mathrm{L}}$ (a) Porosity, (b) permeability, and (c) contact angle. the A-PTL in a PEM electrolyser was derived. This model predicts the saturations and pressure profiles along the spatial coordinate through the transport layer.

In order to solve the model equations a discretisation using a finite volume scheme is provided. The resulting system of equations leads either to a spatially distributed steady-state profile of the saturation and the pressure or to a drying-out effect at the catalyst within finite time.

Hence, a critical current density was computed which separates the steady-state regime from the drying-out regime in terms of current density and channel saturation. It was shown that the material properties of the PTL, i. e. porosity, permeability, and contact angle, have significant influence on the transport limitations of the system, although, the major quantity to prevent a drying-out behaviour is a sufficient water supply in the anode channel of the PEM electrolyser.

Additionally, the properties of a Ti felt which is used as A-PTL in our lab was characterized with regard to its physical properties. Based on the given set of properties, the porosity, the permeability, and the contact angle were varied in three studies in order to show the influence of these three quantities. It can be seen that high porosity, high permeability, as well as low contact angle lead to low transport resistances. Therefore, a suitable material for the anodic PTL sould fulfil the following material properties: porosity $\varepsilon_{0}>0.6$, permeability $k_{0}>10^{-14} \mathrm{~m}^{2}$, and contact angle $\theta<45^{\circ}$. Although it can be seen that a high channel saturation is beneficial in every case, i. e. $S_{\mathrm{ch}}^{\mathrm{L}}>0.8$.

Acknowledgements. The financial support by the German Research Foundation (Deutsche Forschungsgemeinschaft; DFG) within the framework of the project grant SU 189/7-1 is gratefully acknowledged. Furthermore, the authors acknowledge support by the Max Planck Society via the MaxNet Energy research network. 


\section{References}

[1] A. Buttler, H. Spliethoff, Current Status of Water Electrolysis for Energy Storage, Grid Balancing and Sector Coupling via Power-to-Gas and Power-to-Liquids: A Review, Renewable and Sustainable Energy Review 82 (3) (2018) 2440-2454. doi:10.1016/j.rser.2017.09.003

[2] A. H. A. Rahim, A. S. Tijani, S. K. Kamarudin, S. Hanapi, An overview of polymer electrolyte membrane electrolyzer for hydrogen production: Modeling and mass transport, Journal of Power Sources 309 (2016) 56-65. doi:10.1016/j.jpowsour.2016.01.012

[3] A. Awasthi, K. Scott, S. Basu, Dynamic modeling and simulation of a proton exchange membrane electrolyzer for hydrogen production, International Journal of Hydrogen Energy 36 (2011) 14779-86. doi:10.1016/j.ijhydene.2011.03.045.

[4] Z. Abdin, C. J. Webb, E. M. Gray, Modelling and simulation of a proton exchange membrane (PEM) electrolyser cell, International Journal on Hydrogen Energy 40 (2015) 13243-57. doi:10.1016/j.ijhydene.2015.07.129.

[5] T. Yigit, O. F. Selamet, Mathematical modeling and dynamic Simulink simulation of highpressure PEM electrolyzer system, International Journal of Hydrogen Energy 41 (2016) 13901-14. doi:10.1016/j.ijhydene.2016.06.022

[6] J. Nie, Y. Chen, Numerical modeling of threedimensional two-phase gas-liquid flow in the flow field plate of a PEM electrolysis cell, International Journal of Hydrogen Energy 35 (2010) 3183-97. doi:10.1016/j.ijhydene.2010.01.050.

[7] A. C. Olesen, C. Rømer, S. K. Kær, A numerical study of the gas-liquid, two-phase flow maldistribution in the anode of a high pressure PEM water electrolysis cell, International Journal of Hydrogen Energy 41 (2016) 5268. doi:10.1016/j.ijhydene.2015.09.140

[8] E. Arbabi, H. Montazeri, R. Abouatallah, R. Wang, A. Bazylak, Three-Dimensional Computational Fluid Dynamics Modelling of Oxygen Bubble Transport in Polymer Electrolyte Membrane Electrolyzer Porous Transport Layers, Journal of The Electrochemical Society 163 (11) (2016) F3062-9. doi:10.1149/2.0091611jes

[9] S. S. Lafmejani, A. C. Olesen, S. K. Kær, VOF modelling of gas-liquid flow in PEM water electrolysis cell micro-channels, International Journal of Hydrogen Energy 42 (2017) 16333-44. doi:10.1016/j.ijhydene.2017.05.079

[10] H. Kim, M. Park, K. S. Lee, One-dimensional dynamic modeling of a high-pressure water electrolysis system for hydrogen production, International
Journal of Hydrogen Energy 38 (2013) 2596-609. doi:10.1016/j.ijhydene.2012.12.006

[11] F. Aubras, J. Deseure, J.-J. A. Kadjo, I. Dedigama, J. Majasan, B. Grondin-Perez, J.-P. Chabriat, D. J. L. Brett, Two-dimensional model of low-pressure PEM electrolyser: Two-phase flow regime, electrochemical modelling and experimental validation, International Journal of Hydrogen Energy 42 (2017) 26203-16. doi:10.1016/j.ijhydene.2017.08.211

[12] E. T. Ojong, J. T. H. Kwan, A. Nouri-Khorasani, A. Bonakdarpour, D. P. Wilkinson, T. Smolinka, Development of an experimentally validated semi-empirical fully-coupled performance model of a PEM electrolysis cell with 3-D structured porous transport layer, International Journal of Hydrogen Energy 42 (2017) 25831-47. doi:10.1016/j.ijhydene.2017.08.183

[13] A. Nouri-Khorasani, E. T. Ojong, T. Smolinka, D. P. Wilkinson, Model of oxygen bubbles and performance impact in the porous transport layer of PEM water electrlysis cells, International Journal of Hydrogen Energy 42 (2017) 28665-80. doi:10.1016/j.ijhydene.2017.09.167

[14] B. Han, J. Mo, Z. Kang, F.-Y. Zhang, Effects of membrane electrode assembly properties on two-phase transport and performance in proton exchange membrane electrolyser cells, Electrochimica Acta 188 (2016) 31726. doi:10.1016/j.electacta.2015.11.139

[15] B. Han, J. Mo, Z. Kang, G. Yang, W. Barnhill, F.-Y. Zhang, Modelling of two-phase transport in proton exchange membrane electrolyzer cells for hydrogen production, International Journal of Hydrogen Energy 42 (2017) 4478-89. doi:10.1016/j.ijhydene.2016.12.103

[16] C. Immerz, B. Bensmann, P. Trinke, M. Suermann, R. Hanke-Rauschenbach, Local Current Density and Electrochemical Impedance Measurements within $50 \mathrm{~cm}$ Single-Channel PEM Electrolysis Cell, Journal of The Electrochemical Society 165 (16) (2018) F12929. doi:10.1149/2.0411816jes

[17] R. B. Bird, W. E. Stewart, E. N. Lightfoot, Transport Phenomena, John Wiley \& Sons, 2007.

[18] Z. H. Wang, C. Y. Wang, K. S. Chen, Two-Phase Flow and Transport in the Air Cathode of Proton Exchange Membrane Fuel Cells, Journal of Power Sources 94 (1) (2001) 40-50. doi:10.1016/S0378-7753(00)00662-5

[19] M. C. Leverett, Capillary Behaviour in Porous Solids, Transactions of the AIME 142 (1) (1941) 152-169. doi:10.2118/941152-G

[20] N. Zamel, X. Li, A Parametric Study of Multi-phase and Multi-species Transport in the Cathode of PEM Fuel Cell, International Journal of Energy Research 32 (2008) 698-721. doi:10.1002/er.1384 
[21] R. Lemoine-Nava, R. Hanke-Rauschenbach, M. Mangold, K. Sundmacher, The gas diffusion layer in polymer electrolyte membrane fuel cells: A process model of the two-phase flow, International Journal of Hydrogen Energy 36 (2) (2011) 1637-53. doi:10.1016/j.ijhydene.2010.10.037.

[22] S. A. Grigoriev, A. A. Kalinnikov, P. Millet, V. I. Porembsky, V. N. Fateev, Mathematical modeling of high-pressure PEM water electrolysis, Journal of Applied Electrochemistry 40 (5) (2010) 921-32. doi:10.1007/s10800-009-0031-z

[23] L. An, T. S. Zhao, Z. H. Chai, P. Tan, L. Zeng, Mathematical modeling of an anion-exchange membrane water electrolyzer for hydrogen production, International Journal of Hydrogen Energy 39 (2014) 1986976. doi:10.1016/j.ijhydene.2014.10.025

[24] R. Brooks, T. Corey, Hydraulic properties of porous media, Hydrology Papers, Colorado State University 24 (1964).

[25] M. T. van Genuchten, A closed-form equation for predicting the hydraulic conductivity of unsaturated soils, Soil Science Society of America Journal 44 (1980) 8928. doi:10.2136/sssaj1980.03615995004400050002x

[26] R. J. LeVeque, Finite Volume Methods for Hyperbolic Problems, Cambridge University Press, 2002. doi:10.1017/CB09780511791253

[27] E. W. Weisstein, CRC Concise Encyclopedia of Mathematics, CRC Press, 1999.

[28] S. V. Patankar, Numerical Heat Transfer and Fluid Flow, McGraw-Hill, 1980.

[29] L. F. Shampine, M. W. Reichelt, The MATLAB ODE Suite, SIAM Journal on Scientific Computing 18 (1) (1997) 1-22. doi:10.1137/S1064827594276424

[30] J. R. Rumble, CRC Handbook of Chemistry and Physics, CRC Press, 2017.

[31] M. Carmo, D. L. Fritz, J. Mergel, D. Stolten, A comprehensive review on PEM water electrolysis, International Journal of Hydrogen Energy 38 (12) (2013) 4901-34. doi:10.1016/j.ijhydene.2013.01.151.

[32] M. Suermann, K. Takanohashi, A. Lamibrac, T. J. Schmidt, F. N. Büchi, Influence of Operating Conditions and Material Properties on the Mass Transport Losses of Polymer Electrolyte Water Electrolysis, Journal of The Electrochemical Society 164 (9) (2017) F97380. doi:10.1149/2.13517109jes

[33] L. Zielke, A. Fallisch, N. Paust, R. Zengerle, S. Thiele, Tomography Based Screening of Flow Field / Current Collector Combinations for PEM Water Electrolysis, RSC Advances 4 (2014) 58888-94. doi:10.1039/C4RA12402B

[34] A. Z. Weber, J. Newman, Effects of Microporous Layers in Polymer Electrolyte Fuel Cells, Journal of
The Electrochemical Society 152 (4) (2005) A677-88. doi:10.1149/1.1861194

[35] C. Immerz, M. Paidar, G. Papakonstantinou, B. Bensmann, T. Bystron, T. Vidakovic-Koch, K. Bouzek, K. Sundmacher, R. Hanke-Rauschenbach, Effect of the MEA Design on the Performance of PEMWE Single Cells with Different Sizes, Journal of Applied Electrochemistry 48 (6) (2018) 701-11. doi:10.1007/s10800-018-1178-2 